\title{
Filigrane
}

Écoutes psychanalytiques

\section{Temporalité psychique des premières rencontres et noyaux inconscients transmis dans la cure. La fonction-conservatoire des entretiens préliminaires}

\section{Isabelle Lasvergnas}

Volume 27, numéro 2, 2018

La consultation psychanalytique aujourd'hui, entre héritages et remaniements

URI : https://id.erudit.org/iderudit/1055750ar

DOI : https://doi.org/10.7202/1055750ar

Aller au sommaire du numéro

Éditeur(s)

Revue Santé mentale au Québec

ISSN

1192-1412 (imprimé)

1911-4656 (numérique)

Découvrir la revue

Citer cet article

Lasvergnas, I. (2018). Temporalité psychique des premières rencontres et noyaux inconscients transmis dans la cure. La fonction-conservatoire des entretiens préliminaires. Filigrane, 27(2), 69-80.

https://doi.org/10.7202/1055750ar
Résumé de l'article

Le propos théorique de ce texte s'attache à la fonction psychique conservatoire qu'opèrent plus ou moins sourdement chez le patient les entretiens préliminaires sous-tendus par le dispositif d'accueil qui y est offert et son enveloppe psychico-physique. Un exemple clinique permettra d'illustrer la temporalité inconsciente mobilisée/immobilisée chez une patiente lors de ces rencontres inaugurales, et les condensations mnésiques primaires inscrites dans le support transférentiel associé qu'a représenté pour la patiente le double cadre du centre de traitements psychanalytiques. 


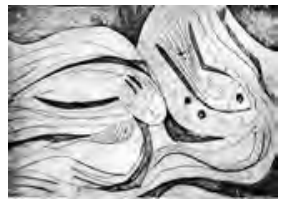

\title{
Temporalité psychique des premières rencontres et noyaux inconscients transmis dans la cure. La fonction-conservatoire des entretiens préliminaires
}

Isabelle Lasvergnas

\begin{abstract}
Résumé: Le propos théorique de ce texte s'attache à la fonction psychique conservatoire qu'opèrent plus ou moins sourdement chez le patient les entretiens préliminaires sous-tendus par le dispositif d'accueil qui y est offert et son enveloppe psychico-physique. Un exemple clinique permettra d'illustrer la temporalité inconsciente mobilisée/immobilisée chez une patiente lors de ces rencontres inaugurales, et les condensations mnésiques primaires inscrites dans le support transférentiel associé qu'a représenté pour la patiente le double cadre du centre de traitements psychanalytiques.
\end{abstract}

Mots clés: dispositif clinique; cadre thérapeutique; double cadre; enveloppe psychique; fonction psychique conservatoire; support transférentiel associé; entretiens préliminaires.

\begin{abstract}
The theoretical aim of this text is tied to the conservatory psychic function that the preliminary clinical meetings, underlain by the setting offered for receiving patients and its physico-psychic envelope character, effect more or less silently in the patient. A clinical example illustrates the unconscious temporality mobilised/immobilized in a female patient during these initial encounters and the primary mnesic condensations inscribed in the associated transferential support that the double setting of the centre of psychoanalytic treatment represented for that patient.
\end{abstract}

Keywords: therapeutic setting; double setting; psychic envelope; conservatory psychic function; associated transferential support; preliminary clinical meetings.

'occasion très rare dans le parcours d'un psychanalyste de pouvoir
contribuer à fonder un nouvel organisme psychanalytique lui offre
l'opportunité précieuse de mener un questionnement approfondi sur le legs
d'une méthode clinique, et les corpus théoriques qui la sous-tendent. Cette 
opportunité, et même ce privilège, furent ceux du petit collectif de cliniciens ${ }^{1}$ qui s'était spontanément constitué à l'automne 2006 pour assumer, au nom de la Société psychanalytique de Montréal, la mise en place de la Clinique psychanalytique de Montréal (CPM).

Par-delà les préoccupations et contraintes organisationnelles qui pesaient de leurs poids de réalité empirique et concrète, devoir fonder ce nouveau centre destiné à des patients économiquement et psychiquement vulnérables nous amenait également à reprendre pour notre propre compte les enseignements considérables de plusieurs cliniques à visée sociale dont les travaux et publications avaient largement inspiré la réflexion du comité préparatoire de la CPM entre les années 1996 et $2005^{2}$. Il s'agissait tout particulièrement des expériences de longue date du CCTP Jean-Favreau, du Centre Évelyne et Jean Kestemberg, de la Tavistock Clinic, et des développements novateurs dont ces centres furent l'occasion.

Le travail requis à l'occasion de l'ouverture de la CPM fut une réflexion partagée dans des échanges explicitement formulés entre nous. Mais il fut aussi un travail subjectif de nature auto-analytique qui impliquait chez chacun la ré-interrogation de sa propre compréhension des fondements épistémologiques chez Freud du dispositif fondateur de la cure-type; et, après Freud, des motifs et des conditions des remaniements de ce cadre-princeps que l'approfondissement clinique de plusieurs décennies et l'extension de la pratique à de nouvelles problématiques psychopathologiques et catégories de patients avaient permis d'introduire. Ou pour le dire autrement, le questionnement mené convoquait dans les espaces psychiques subjectifs ainsi que dans l'espace psychique groupal, des représentations plurielles touchant à l'ensemble des éléments constitutifs d'un cadre de traitement que l'on voulait conforme à un idéal du Moi analytique. Une convocation interne et intime d'autant plus intense, et éventuellement d'autant plus déstabilisante, que l'emprise inconsciente de l'idéalisation d'un cadre prototypique n'est pas sans être infiltrée dans la personnalité socioprofessionnelle du psychanalyste de noyaux refoulés hérités de sa propre cure. En bref, il nous incombait subjectivement et groupalement d'adopter une position de recherche clinique: ce qui incombe par principe à tout analyste, mais en l'occurrence d'une manière sans doute plus affirmée et plus intense qu'à l'habituel du quant-à-soi d'un bureau privé, et son caractère d'abri à l'endroit du regard des autres.

S'il me fallait définir en quelques mots ce qu'implique d'un point de vue psychanalytique se mettre en position de recherche clinique, je dirais que 
c'est en premier lieu s'efforcer de se désolidariser de la tentation d'une fusion avec des figures tutélaires «surpuissantes» dont la première configuration provient de l'analyste de l'analyste. C'est s'efforcer de se distancier de mouvements d'identification constitutifs d'un narcissisme groupal, et renoncer au confort du "prêt à penser» que peut conférer l'illusion d'une corporéité fusionnante qui est indistinctement théorico-idéologico-pulsionnelle. En ce sens, fonder la CPM, et y œuvrer, a joué pour l'équipe pionnière à l'analogie d'un effet loupe révélateur des modèles théoriques prédominants chez chaque membre de l'équipe. Révélateur également de sources de résistances et d'écueils transférentiels pouvant alimenter des automatismes de répétition (Anzieu, 1976).

En termes de méthode, les analystes s'accordent sur l'importance de l'analyse des mouvements transférentiels dont la relation patient-thérapeute est le moteur et le support, de même que sur le caractère éminemment privé de cette relation. Mais dans le cas des traitements menés dans un organisme clinique, il y a désaccord, et même carrément opposition d'écoles, quant à la pertinence d'accorder une attention particulière à ce que je nommerai la fonction transférentielle associée $e^{3}$ occupée par l'organisme en tant que «sur-cadre» du cadre formel de traitement et de la rencontre transféro-contre-transférentielle.

Un tel désaccord était présent au sein même de l'équipe pionnière de la CPM. S’y exprimaient des écarts sensibles dans la conception de l'éthique de la pratique, et dans le degré de prééminence à accorder au désir - inconscient - de l'analyste (Lacan, 1986) dans sa décision de s'engager avec un patient et de déterminer les conditions de sa cure.

D'un côté, on considérait que l'instance organisationnelle empirique d'un centre de consultation constitue une impureté du cadre dont il faut s'efforcer de rendre le plus inopérantes possible les incidences éventuellement négatives dans la dynamique de la cure, voire ignorer ou effacer dans l'écoute prêtée au patient l'ombre de sa présence et ses valences inconscientes.

De l'autre, si on reconnaissait sans réserve l'importance de préserver la relation transféro-contre-transférentielle des risques d'une ingérence administrative désymbolisée et en surplomb, et potentiellement effractive, on estimait que la réalité même du centre clinique fait de celui-ci partie constitutive du cadre de traitement, et qu'on ne pouvait dès lors lui attribuer le statut d'instance inexistante et non-signifiante dans les processus de la cure.

Ma position théorique personnelle était largement redevable aux apports de Paul Racamier, Évelyne Kestemberg, André Green et Jean-Luc Donnet sur 
le caractère primordial de la structuration du cadre de soin dans un organisme clinique, et la fonction symbolique tiercéisante tenue par l'organisme dans la dualité du couple patient-thérapeute - fonction particulièrement cruciale dans le cas des états limites et psychotiques. Dans leur mise en évidence, tour à tour, des fonctions symboliques de rempart contre-incestuel imaginairement attribué par le patient à l'organisme clinique (Racamier, 1970), de point d'appui en la personne incarnée du directeur du centre de traitements dans le cas des problématiques psychotiques (Kestemberg, 1981 ) et de différenciation des temporalités entre entrevues de «consultation» et prise en charge du traitement proprement dit (Donnet, 2005), les élaborations proposées m'apparaissaient incontournables. - On sait que dans ces deux derniers cas, les principes théoriques dégagés ont trouvé leurs traductions dans les structures organisationnelles mises en place au Centre Kestemberg et au CCTP Jean-Favreau (personnage-tiers et double cadre consultation/traitement) ${ }^{4}$.

Je situerai ma propre élaboration dans le prolongement de ces positions théoriques, en m'attachant plus particulièrement à une dimension transférentielle projetée sur l'organisme clinique peu ou non développée jusqu'ici: soit la potentialité mnésique tenue par la double enveloppe psychique qui est offerte à un patient lorsque celui-ci est rencontré dans un centre de traitements. Je nommerai fonction psychique conservatoire la fonction opérée de manière plus ou moins manifeste par les premières rencontres avec un patient dans leur conjugaison de temporalité psychique et de dispositif formel d'accueil: dans le cas spécifique de la CPM qui servira de point d'appui à ma propre proposition, il s'agit d'un dispositif plurisubjectif ${ }^{5}$ originairement mis en place par le collectif pionner de l'organisme, dans un mouvement que j'ai qualifié de «trouvé/créé» collectif, et qui s'est révélé à l'expérience d'une grande richesse clinique (Lasvergnas, 2014, 2018).

De brefs extraits de séances avec une patiente me serviront d'illustration.

Il s'agit d'une jeune femme à la fin de la trentaine, alors dans la cinquième année d'une thérapie psychanalytique menée en face à face. Nous envisagions à ce moment depuis environ six mois la perspective d'intensifier le nombre des séances hebdomadaires pour mettre en place un cadre de cure analytique plus «classique», ce que la patiente vivait dans une profonde ambivalence marquée d'hésitations, d'anxiétés paranoïdes et de nombreux allers-retours dans sa prise de décision.

L'événement psychique que j'évoquerai dans le cas de cette patiente fut un moment cristallisateur de sa problématique transférentielle négative, et 
de deux modalités nodales de ses relations avec les objets primaires. Un tel événement s'est produit au moment de la reprise de mes consultations après mes vacances d'été.

Pendant un mois, Ophélia ne s'est pas présentée à son rendez-vous hebdomadaire. Ce genre d'absences prolongées sans le moindre préavis avait été un des symptômes dominants tout au long de la troisième année de la cure. Abondamment travaillées en thérapie, les absences avaient peu à peu cessé. Ces périodes d'absences, je l'avais progressivement appris, s'accompagnaient d'un retrait social marqué de la jeune-femme qui était alors "engloutie", selon ses propres mots, dans une angoisse insurmontable, sinon à la noyer dans l'alcool, tout en étant taraudée par la pensée que j'allais à tout moment lui annoncer qu'il était mis fin à la thérapie partiellement subventionnée dont elle bénéficiait, et dont elle s'absentait trop souvent ${ }^{6}$. Progressivement, compte tenu de l'intensité des affects de détresse qui s'emparaient d'Ophélia dans ces périodes où elle disparaissait physiquement, j'avais mis en place une sorte de rituel entre nous suivant lequel, au bout de deux ou trois semaines d'absence, je prenais l'initiative de "lui parler», c'est-à-dire que je lui laissais un bref message téléphonique (son téléphone dans ces périodes-là était uniquement sur répondeur) lui disant que comme elle le savait, je l'avais attendue, que j'étais là, et que je l'attendais la semaine suivante à l'heure habituelle de son rendez-vous. Il m'est arrivé de devoir répéter ces messages jusqu'à trois ou quatre semaines consécutives. Lorsqu'enfin Ophélia reprenait le rythme de ses séances, elle s'acquittait scrupuleusement du règlement de celles qu'elle avait manquées, tout en m'en demandant, à chaque fois leur nombre.

Lorsqu'il advint dans la cure que j'eus à lui faire part de mon retrait de la Clinique psychanalytique de Montréal, elle choisit spontanément de continuer sa démarche psychothérapique avec moi dans un contexte de pratique libérale ${ }^{7}$. "Je reste avec vous» m'avait-elle dit laconiquement, sans porter en apparence davantage d'intérêt à ce changement majeur dans le cadre clinique, et sans que j'aie pu par la suite - j’y étais très attentive - déceler, y compris dans des rêves, une trace significative de l'impact en elle de cet événement. Comme si le maintien du lien personnalisé avec moi avait été la seule chose qui importait pour elle à ce moment-là.

Lorsqu'au début de l'automne Ophélia se présenta à nouveau à sa séance hebdomadaire après un appel téléphonique de ma part, et alors que près de quinze mois s'étaient écoulés depuis la modification/rupture du cadre antérieur intervenue de mon fait, elle apporta l'explication suivante: «Je ne me 
souvenais plus de la date de votre retour, je pensais avoir perdu votre numéro de téléphone, et comme vous m'aviez dit que vous n'alliez plus être membre de la Société de psychanalyse, je me disais que je ne pouvais plus trouver votre nom nulle part, et que je ne pouvais plus vous appeler pour vérifier». Tout en ajoutant avec nuance et finesse: «j'étais convaincue d'avoir perdu votre numéro de téléphone; en même temps, quelque chose en moi me disait que je me trompais, et que si je le cherchais, je le retrouverais. Mais c'est ma conviction de l'avoir perdu qui l'emportait».

Si dans cette séquence interrelationnelle l'état de déroute qu'avait produit chez Ophélia l'éprouvé d'une disparition physique de ma part pendant mon congé estival était indubitable - autant que l'était la dimension de castration de l'objet transférentiel qui perdait un de ses attributs identitaires se révélait aussi, et c'est sur ce point que j'insisterai, une autre composante du transfert de fond reliée au cadre de traitement. Reliée au cadre du cadre en l'occurrence. Soit le dépôt inconscient qui s'était logé dans le méta-dispositif représenté par «La Clinique», et dans lequel s'insérait le cadre de la rencontre patient/thérapeute, soit, dans ce cas, une rencontre en face à face, une fois par semaine.

Dans ce nouvel acting de répétition d'absence, il me semblait que se révélait la nature du retentissement qu'avait eu pour la patiente le fait de mon détachement institutionnel de la CPM. S'y réactivait en l'occurrence, plus clairement que nous n'avions pu l'analyser jusqu'ici, une des modalités prédominantes de sa relation à l'objet maternel.

De fait, son attachement affectif apparent à sa thérapeute dans sa décision précipitée de continuer avec moi son traitement avait relevé plutôt de l'ordre d'un agrippement à une mère vécue depuis toujours sous menace de disparition physique pour l'enfant.

Contigu à l'oubli de la date de reprise de mes consultations, et au désancrage chez Ophélia d'avec une certaine réalité d'un temps chronologique lors de chacune de ses absences, se dévoilait ici un large pan de la fonctionholding silencieusement tenue par l'institution CPM dans laquelle s'était inauguré le traitement entrepris. Je parlerai plus spécifiquement dans ce cas d'une fonction de fixation spatio-temporelle de l'objet maternel. Fixation précaire je croirais, à peine plus corporéisée peut-être qu'un certificat de dépôt dans une consigne de gare ou l'inscription d'un nom dans un bottin professionnel, mais me permettant toutefois d'entendre le fantasme dans lequel j'avais perdu un des signes de mon identité socio-professionnelle, à l'analogie de l'écriture d'un rêve. J'y décelais l'amorce d'un processus de 
traduction préconsciente d'une angoisse longtemps demeurée hors les mots, et reliée à une faille dans la partie identitaire maternelle qui m'était attribuée dans le transfert, et par rapport à laquelle l'enveloppe contenante de la CPM faisait office de contrepoids et de garant de continuité.

Jusqu'ici, la jeune femme avait donc oscillé dans sa cure entre une présence/absence physique à ses séances et la détresse induite par un non-là de l'objet maternel, qui n'était pas tant une absence radicale de l'objet, qu'une incertitude à pouvoir le trouver/retrouver. Un vide dans la psyché infantile de la capacité d'internalisation d'une présence suffisamment stable, et suffisamment contenante et attentive aux besoins de l'enfant. S'il me fallait chercher une équivalence métaphorique pour traduire l'éprouvé qui m'était communiqué, j'évoquerais l'énigme qui a sous-tendu les recherches sur les champs magnétiques de Maxwell et qui lui ont permis d'aboutir à ses équations mathématiques sur un vide qui contient de la matière.

Je comprenais également que le surmoi paternel censeur et punitif qui m’avait été attribué lors des périodes précédentes d'absences récurrentes chez Ophélia, l'avait été au nom de ma fonction de déléguée de « $L a$ Clinique» comme elle nommait cet organisme, sans doute à partir de mes propres mots $^{8}$. Cette fonction-tiers paternelle autoritaire et menaçante, fondue dans l'existence matérielle du cadre du cadre, avait néanmoins le mérite d'endiguer des angoisses massives liées à une inconstance de la présence, attribuée dans le discours manifeste et dans le transfert à la personnalité de la mère de la patiente.

Dans la répétition de la temporalité psychique illustrée par ce moment précis de la cure, et dans la dissociation des imagos maternelles et paternelles projetées sur le cadre de «La Clinique», s'offrait ainsi à l'analyste une traduction expressive de la double fonction de barrière symbolique que ce cadre représentait: «barrière symbolique, paternelle et sur-moïque et enclos symbiotique et maternel» pour reprendre les qualificatifs de J.-L. Donnet (cité dans Bournova et Kapsembelis, 2005, p. 670). On peut postuler que dans l'après-coup de mon départ de la CPM, Ophélia avait été laissée dans un éprouvé de vacuum et livrée par la figure paternelle, et ses tenant-lieux surmoïques, dans un défaut de repères spatio-temporels à un corps-à-corps mère/infans marqué par une déliaison de la présence maternelle. Ce que je décrivais un peu plus haut, par le paradoxe d'un vide plein.

On pourrait dire de cette vignette clinique qu'elle est exemplaire d'une relative incapacité dans la psyché infantile de symbolisation et d'intériorisation d'une présence/absence de l'objet maternel primaire. Ou encore, qu'elle 
reflète une impasse de la possibilité de figuration d'un quelconque jeu du fort-da, de par l'amputation de la pensée que l'annonce de mon départ de la CPM avait produite chez la patiente, et par rapport à laquelle son empressement à continuer la thérapie avec moi avait joué le rôle de métadéfense contre la sensation physique envahissante d'un danger d'abandon imminent, et d'une fracture dans l'équilibre de l'environnement. De cette teneur de détresse qu'avait éprouvée par exemple l'adolescente au moment de la séparation de ses parents, et de son incertitude depuis toujours quant à la constance de l'investissement affectif dont elle se sentait être l'objet de leur part. Un souvenir-écran donnait à voir une scène de dispute entre les deux parents qui n'auraient voulu ni l'un ni l'autre s'encombrer dans leur nouvelle vie de la charge de leurs enfants.

Nous sommes familiers avec l'apport majeur de Jose Bleger (1979) sur la fonction silencieuse tenue par le cadre clinique sur lequel se fixent les angoisses les plus archaïques, au plus proche d'un noyau psychotique, et qui ne se révèlent que lorsque le cadre "grince» ou se déstabilise, ce qui fut bien le cas dans le parcours de cette cure, et tout particulièrement dans ces mois-là.

Mais j'emprunterai à R. Kaës un autre éclairage de l'alliance thérapeutique inconsciente qui s'était nouée entre ma patiente et moi, et que Kaës qualifierait de "négativité relative», au sens où cette forme de négativité «définit un champ du possible (et) soutient l'espace potentiel de la réalité psychique». Je le cite:

La négativité relative définit un autre espace et une autre expérience psychique. Le négatif concerne ici ce qui ne s'est pas réalisé dans la réalité psychique, ce qui est resté en souffrance dans la formation des contenants et des contenus psychiques, ce qui n'a pas eu lieu ou n'a pas trouvé de lieu ou de moyens. [...] L'objet et l'expérience de l'objet ont été constitués, mais leur disparition, leur insuffisance, leur défaut, laisse ouverte une attente, un désir. (Kaës, 2014, p. 108)

Dans la séance qui a suivi, Ophélia a évoqué les rencontres préliminaires qui s'étaient tenues près de cinq ans plus tôt à l'époque. Leur souvenir lui en est revenu comme celui d'un espace de brume et d'angoisse dans lequel elle se sentait perdue, une sensation physique invasive et récurrente dans son enfance, de l'ordre de la terreur/fureur d'un Petit Poucet abandonné au fond des bois. Se souvenant de la trace en elle de la présence des analystes 
évaluateurs lors de ces rencontres, Ophélia évoquera des silhouettes grises, sans couleur, indistinctes. Puis elle se mettra à pleurer doucement. C'était une des toutes premières fois où elle pleurait pendant une séance. Par contraste avec l'écriture mémorielle de cette scène clinique inaugurale, elle évoquera immédiatement des amis qu'elle a. J'ai eu à cet instant précis la sensation puissante que cette évocation qui surgissait chez elle était une tentative forcenée de se raccrocher à une bouée psychique, n'importe laquelle.

- Ce sont des vrais fous... me dira-t-elle à plusieurs reprises. Vous savez, des fous, vraiment des fous, mais ils ont de la couleur eux, ils ont du panache.

— Comme votre mère! ai-je dit.

Déstabilisée quelques secondes, interloquée par cette vision inattendue qui lui était restituée de sa mère, Ophélia acquiescera presque joyeuse, presque consolée sur l'instant: "Oui, c'est vrai, ma mère, elle, elle a du panache!»

Puis elle reviendra sur le gris et sur la souffrance "d'une solitude intolérable» qui la plonge en permanence «dans un gouffre de grisaille». C'est alors que pour la première fois dans la cure a surgi un dédoublement de la figure paternelle ${ }^{9}$, et que derrière la façade d'un père rigide et inflexible, à qui il était difficile de communiquer ses émotions intimes, ce contre quoi se déchainait la rage d'Ophélia, a surgi la tristesse du père. Ophélia dira mot à mot: "Je me rends compte qu'il y a à l'intérieur de mon père une carapace dans laquelle il est emprisonné, et il ne le sait pas. Je me rends compte que j'ai su depuis toujours que mon père était quelqu'un d'infiniment triste». Après un très long silence, elle ajoutera: «Il y a en moi la douleur d'une solitude terrifiante. Terrifiante et insupportable, pas seulement pour moi, mais qui le serait pour n'importe qui. C'est une douleur enracinée dans mon corps, je ne peux pas la ressentir, c'est corporellement bloqué. Je ne peux l'approcher que si je la reconnais chez quelqu'un d'autre. Je ne pourrais l'éprouver qu'à travers un autre.»

Le dispositif plurisubjectif de la consultation mis en place à la CPM en 2007 au moment où furent reçus les premiers patients, et qui est repris aujourd'hui dans le GPMR, a l'immense mérite, en donnant à voir «une sorte de diffraction expérimentale du registre contre-transférentiel» (Donnet, 2018, inédit), de mettre en jeu dans un groupe de psychanalystes 
rassemblés une expérience intersubjective de l'inconscient difficilement accessible autrement.

La pratique de ce dispositif depuis plus de dix années maintenant nous a également permis de repérer du côté des patients l'apparition rapide de configurations archaïques de liens qui en général ne se font jour que beaucoup plus lentement dans un dispositif duel patient/thérapeute. Selon les cas, il s'agira de mobilisations de représentations latentes relativement à bas bruit, ou au contraire de manifestations psychiques très puissantes; par exemple lorsque dans les rencontres préliminaires se produit une forte mobilisation de la part psychotique de la personnalité et qu'apparaissent d'entrée de jeu des modalités de relations d'objet empreintes de mécanismes projectifs paranoïdes ou au contraire d'une quête fusionnelle, accompagnés de mouvements pré-transférentiels diffractés et clivés entre les divers thérapeutes présents, incluant l'organisme clinique lui-même.

Le retour dans une perspective historique sur le développement de la théorisation de la cure, nous rappelle que, dans un premier temps, Freud et les premières générations d'analystes se sont attardés aux contenus inconscients, fantasmes refoulés, souvenirs-écrans et conflictualités intrapsychiques en lien avec les objets internes. Mais l'extension de la pratique avec les enfants, les états limites, les psychotiques, les familles et les groupes ont contraint les analystes à s'intéresser de plus en plus aux structures contenantes et à leurs fonctions.

À propos de leur travail dans les centres de consultations psychanalytiques, Donnet et Green écrivaient dès 1973, et dans le prolongement de leur notion de psychose blanche, que le travail dans ces centres a contribué à introduire un déplacement dans l'écoute du clinicien de «l'analyse de contenu [...] à l'analyse de contenant, à savoir du cadre, et de ce qui dans l'activité de pensée, permet qu'une telle activité puisse être déployée» (cité dans Bournova et Kapsembelis, 2005, p. 671).

C'est ce dont à mon tour j'ai visé à témoigner dans l'approfondissement de ma réflexion sur le double cadre à partir d'un exemple de répétition inconsciente dans la relation transférentielle, dans laquelle le cadre du cadre, à titre de sur-enveloppe du cadre relationnel de la cure, avait agi comme l'instance silencieuse d'une mise en abyme des deux figures parentales. Et ultimement, comme le réceptacle-conservatoire et le premier contenant mnésique d'une identification infantile à un noyau mélancolique du père.

Pour l'exprimer d'une manière plus générale et mieux spécifier le point théorique que je veux faire valoir, je dirais que l'écoute portée dans le 
déroulement des traitements à la temporalité inconsciente qui avait pu être mobilisée/immobilisée chez les patients lors des rencontres préliminaires m'a permis de repérer une ligne de force intemporelle, le plus souvent faite d'éprouvés ancrés dans le corporel - ce que le dispositif des rencontres de consultation à la CPM ou au GPMR sollicite et active sans doute davantage que celui, plus habituel, dans une pratique en bureau privé.

Comme dans le cas particulier d'Ophélia, et plus généralement dans le cas des problématiques limites et psychotiques, il s'agit de la cristallisation d'éprouvés bruts et de noyaux pré-imagoïques «impassés» (Scarfone, 2014). Une empreinte s'esquisse provisoirement atteinte par le refoulement ou le clivage. Elle est sédimentée. Elle recouvre et condense de puissants mouvements d'affects inconscients et des mouvements pré-transférentiels diffus et déjà là qui seront maintenus longtemps dans la cure, non-inscrits dans un contexte représentatif lisible ou devinable.

Ces condensations mnésiques primaires apparentées à l'écriture scripturale d'un bloc-notes magique sont déposées dans le support transférentiel associé que le dispositif des rencontres de consultation dans un cadre institutionnel - en tant que métonymie de l'organisme clinique et en tant que sa première manifestation d'existence - peut constituer pour le patient. La force d'écriture imaginaire de ces condensations mnésiques se révèle dans leur émergence progressive dans le processus du travail psychothérapique. S’y révèle également la fonction-conservatoire, «de réceptacle et de maintien» dirait A. Ciccone (2001), que tiennent pour les patients les rencontres inaugurales et leur dispositif physico-psychique contenant.

\author{
Isabelle Lasvergnas \\ lasvergnas.isabelle@uqam.ca
}

\title{
Notes
}

1. Ce groupe était constitué par Marie Claire Lanctôt Bélanger, Monique Meloche, Louis Pinard, Micheline Reid-Perreault et moi-même.

2. J'ai assumé après Louise Quintal la coordination de ce comité de 2000 à 2005. J'ai eu l'honneur d'occuper par la suite les fonctions de directrice de la CPM de 2006 à 2014.

3. Mes termes.

4. Le lecteur pourra se référer aux textes d'Alain Gibeault et Danielle Kaswin dans la présente monographie. Voir également le numéro thématique «Le tiers institutionnel» (2018), dans Psychanalyse et psychose, $\mathrm{n}^{\circ} 18$.

5. Pour des raisons dictées par le manque de moyens financiers et de possibilité de location d'un local identificateur du site de la CPM, faute de murs contenants pour le patient, et dans la nécessité d'inscrire la réalité tiercéisante de la CPM dans la prise en charge des traitements offerts, ainsi que dans celle de créer entre les thérapeutes un espace 
de réflexion clinique partagé et un espace de recherche commune, l'équipe clinique fondatrice de la CPM a inventé un dispositif de consultation plurisubjectif pour les entretiens préliminaires. Les patients sont reçus par des équipes en principe de trois analystes-consultants dans des locaux loués à l'heure. Suite à ces premières entrevues d'un minimum de deux, pouvant aller jusqu'à trois, voire quatre dans certains cas, les patients seront par la suite reçus dans le bureau privé de l'analyste en charge du traitement. La contribution monétaire du patient au coût de son traitement partiellement subventionné sera toujours faite au nom de la CPM. Ce même dispositif de double cadre a été adopté au Groupe psychanalytique du Mont-Royal (GPMR). La mise en place de ce dispositif qui fut au départ un geste pragmatique et a-théorique faisait appel nécessairement à sa théorisation progressive.

6. La démarche psychothérapique d'Ophélia avait été entreprise dans le cadre de la CPM.

7. Plutôt que de changer de psychothérapeute en restant une patiente de la CPM, possibilité qui lui fut offerte en lui laissant quelques semaines pour pouvoir élaborer minimalement le choix qui serait le sien.

8. Chaque fois que pertinent, j'étais très attentive à rappeler aux patients que je recevais en consultation dans mon bureau privé, mais dont le traitement relevait de la CPM, l'instance tierce représentée par la Clinique. Par exemple, au moment du règlement des séances, je remerciais en nommant la Clinique «à qui je vais transmettre ce règlement, etc.» J'agis de même dans le cadre du GPMR.

9. Ce dédoublement s'est produit à partir d'une construction de ma part, étayée sur la réminiscence associative en moi à cet instant précis de la séance, d'un souvenir-écran relaté par Ophélia lors de la première des trois rencontres évaluatives qui avaient eu lieu dans son cas. Cette figuration mémorielle chez la patiente avait été particulièrement expressive d'une détresse infantile dans un lien privilégié avec l'objet paternel.

\section{Références}

Anzieu, D. (1976). La fantasmatique de la formation psychanalytique. Repris dans R. Kaës et al. (2001), Fantasme et formation. Paris: Dunod

Bleger, J. (1979). Psychanalyse du cadre psychanalytique. Dans D. Anzieu, R. Kaës et al., Crise, rupture et dépassement (p. 255-285). Paris: Dunod.

Bournova, K. et Kapsembelis, V. (2005). Argument. Revue française de psychanalyse, 69 (3), 669-674.

Ciccone, A. (2001). Enveloppe psychique et fonction contenante: modèles et pratiques. Cahiers de psychologie clinique, 17 (2), 81-102.

Donnet, J.-L. (2005). La situation analysante. Paris: Presses universitaires de France.

Kaës, R. (2014). Les alliances inconscientes. Paris: Dunod.

Lacan, J. (1986). Le Séminaire VII, L'éthique de la psychanalyse (1959-1960). Paris: Le Seuil.

Kestemberg, É. (1981). Le personnage tiers. Sa nature, sa fonction. Dans auteur (2001), La psychose froide (p. 144-147). Paris: Presses universitaires de France.

Lasvergnas, I. (2018). Naissance d'une clinique psychanalytique: entre héritages transférentiels et espace transitionnel partagé. Le Carnet-Psy, 217, 44-49.

Lasvergnas, I. (2014). Les restes-toujours-à-penser d'une filiation: À propos du cadre clinique. Canadian Journal of Psychoanalysis/Revue canadienne de psychanalyse, 22 (1), 66-84.

Racamier, P. C. (1970). Le psychanalyste sans divan. Paris: Payot.

Scarfone, D. (2014). L'impassé, actualité de l'inconscient. Revue française de psychanalyse, 78 (5), 1357-1428. 